\section{Near-Infrared Characterization of Breast Tumors In Vivo using Spectrally-Constrained Reconstruction}

Www.tcrt.org

Multi-wavelength Near-Infrared (NIR) Tomography was utilized in this study to non-invasively quantify physiological parameters of breast tumors using direct spectral reconstruction. Frequency domain NIR measurements were incorporated with a new spectrally constrained direct chromophore and scattering image reconstruction algorithm, which was validated in simulations and experimental phantoms. Images of total hemoglobin, oxygen saturation, water, and scatter parameters were obtained with higher accuracy than previously reported. Using this spectral approach, in vivo NIR images are presented and interpreted through a series of case studies ( $n=6$ subjects) having differing abnormalities. The corresponding mammograms and ultrasound images are also evaluated. Three of six cases were malignant (infiltrating ductal carcinomas) and showed higher hemoglobin (34-86\% increase), a reduction in oxygen saturation, an increase in water content as well as scatter changes relative to surrounding normal tissue. Three of six cases were benign, two of which were diagnosed with fibrocystic disease and showed a dominant contrast in water, consistent with fluid filled cysts. Scatter amplitude was the main source of contrast in the volunteer with the benign condition fibrosis, which typically contains denser collagen tissue. The changes monitored correspond to physiological changes associated with angiogenesis, hypoxia and cell proliferation anticipated in cancers. These changes represent potential diagnostic indicators, which can be assessed to characterize breast tumors.

\section{Introduction}

Near-Infrared (NIR) imaging of tissue can potentially provide quantitatively accurate estimates of physiologically important parameters such as hemoglobin, oxygen saturation, water fraction, lipid fraction, and scattering indices (1-6). However, past approaches to NIR tomography have suffered from using too few wavelengths, and simplistic strategies for spectral deconvolution. More recently there have been significant breakthroughs in the development of algorithms which incorporate the underlying multispectral constraints available from chromophore extinction spectra within the image reconstruction process (7-10). In this paper, an optimized direct spectral reconstruction technique is implemented and evaluated in phantoms and initial patient studies to demonstrate the potential benefits of this algorithmic approach. The major benefits of direct spectral reconstruction are well established $(9,10)$ and have shown to lead to improvements in the accuracy of quantification of oxygen saturation, water fraction, and scattering parameters. The importance of improved accuracy in these parameters is significant, because they provide fundamental metabolic information about tissue which is not currently being obtained with any other imaging modalities.

The value of oxygen saturation in particular could be significant, if sufficient accuracy can be achieved in estimating this parameter with an imaging method.
Subhadra Srinivasan, Ph.D. ,** $^{*}$

Brian W. Pogue, Ph.D. 1,*

Ben Brooksby, Ph.D.1

Shudong Jiang, Ph.D. ${ }^{1}$

Hamid Dehghani, Ph.D. 1

Christine Kogel, R.N.2

Wendy A. Wells, M.D. ${ }^{3}$

Steven P. Poplack, M.D. ${ }^{4}$

Keith D. Paulsen, Ph.D. ${ }^{1}$

${ }^{1}$ Dartmouth College

Thayer School of Engineering

Hanover, New Hampshire 03755, USA

${ }^{2}$ Dartmouth Hitchcock Medical Center

Norris Cotton Cancer Center

Lebanon, New Hampshire 03756, USA

${ }^{3}$ Department of Pathology

Dartmouth Hitchcock Medical Center

Norris Cotton Cancer Center

Lebanon, New Hampshire 03756, USA

${ }^{4}$ Department of Radiology

Dartmouth Hitchcock Medical Center

Norris Cotton Cancer Center

Lebanon, New Hampshire 03756, USA

* Corresponding Authors:

Subhadra Srinivasan, Ph.D.

Email: subha@dartmouth.edu

Brian W. Pogue, Ph.D.

Email:pogue@dartmouth.edu 
Tissue hypoxia is found in breast cancers due to metabolic imbalance between oxygen supply and consumption (11), which has significant potential in diagnosis as well as prognosis for radiation treatment. On average, the mean partial pressure of oxygen $\left(\mathrm{pO}_{2}\right)$ is lower in malignancies than in surrounding tissues (typically $\leq 20 \mathrm{~mm} \mathrm{Hg}$ ) (11). This presents a difficult situation for radiotherapy which is ineffective when $\mathrm{pO}_{2}$ is less than $5 \mathrm{~mm} \mathrm{Hg}$ (12). Tumor oxygenation may serve to predict its response to radiation treatment as suggested by its critical role in modifying the dose response curve (13) and may also be related to the likelihood of occurrence of distant metastases as well (14). Vaupel et al. (15) have shown that although hypoxia did not correlate with tumor size, tumor location, grade or stage, it did depend critically on whole blood hemoglobin levels; even mild anemia in breast cancer subjects causes the development of hypoxia. Pretreatment hemoglobin levels (16) can also aid in predicting tumor response to primary chemotherapy. While the hemoglobin levels referred to here were baseline values, those measured by NIR imaging for the normal tissue surrounding the tumor may also be representative of the oxygen carrying capacity of the blood in the breast.

Conover et al. (17) used NIR spectroscopic measurements in vivo on subcutaneous rat mammary adenocarcinomas to show correlation with cryospectrophotometry estimates of oxygen saturation on rapidly frozen sections of the same tumors. These studies suggested that NIR may be able to detect hypoxic regions even when their distribution occurs on a spatial scale that is beyond the resolution limit of NIR. Tromberg et al. (18) observed a decrease in oxygen saturation in spectroscopic studies on a palpable mass diagnosed as ductal carcinoma in situ (DCIS). In a related study which monitored neoadjuvant chemotherapy using spectroscopy (19), they found an initial tissue oxygen saturation decrease in the lesion with respect to the surrounding tissue followed by a slight peak, when tracked over a ten week course of treatment. Spectroscopic methods, however suffer from over-sampling of the superficial bulk tissue, which while suitable for palpable tumors, may misrepresent spatial changes in deeper lesions. Tomographic imaging methods sample more broadly and provide a better representation of the whole breast volume. In a study (20) using time domain instrumentation with two wavelengths and a water contribution fixed at $30 \%$, lower oxygen saturation was observed in images of two carcinomas. Heffer et al. (21) used an 'oxygenation index', generated from frequency domain measurements, to show a decrease in carcinomas. In previous work at Dartmouth, McBride et al. (22) used an NIR tomography system to image a subject with a $2.5 \mathrm{~cm}$ infiltrating ductal carcinoma, and although an increase in hemoglobin was observed, reduction in oxygen saturation was not found. Dehghani et al. (23) used three-dimensional modeling to obtain tomographic images from a patient with a infil- trating ductal carcinoma and reported an increase in blood oxygen saturation level, contrary to expectation. Grosenick et al. (24) surveyed results from 50 carcinomas using a dual wavelength time domain instrument and showed good separation of tumors from healthy tissue based on total hemoglobin. However, in their study oxygen saturation both increased and decreased at the location of tumors such that no clear hypoxic trend was evident.

Thus, it appears that without direct spectral reconstruction, the accuracy of predicting oxygen saturation values may be diminished. In this paper, the spectrally constrained direct chromophore and scattering reconstruction which has been shown to be quantitatively superior and stable to measurement and image noise in homogeneous phantom measurements (9) and simulations (25), is validated further in experiments and is applied to the study of breast lesions.

Another NIR parameter of significant interest is scattering. Optical scattering has been correlated to mammographic density (26), which is a major risk factor in the development of cancer (27). A recent study (28) to assess NIR transillumination spectroscopy as related to mammographic density and cancer risk, showed that optical spectroscopy predicted the radiological assessment of density with a principal components analysis (PCA) model in the range of $90 \%$ with an odds ratio comparable to mammography. In breast cancer locations, scattering is certainly expected to increase because the tumor cells stimulate endothelial cell proliferation (29), which increases the cellular density. In addition, the tortuous tumor vessel network is held together by dense fibrotic connective tissue (30), which may be optically dense, owing to the presence of different sized scatterers relative to the surrounding tissue. Spectroscopic studies have shown this increase in scattering in a palpable carcinoma (31). In this paper, scattering is analyzed from the region of the tumor, as detected in the tomographic images obtained through spectrally constrained direct reconstruction.

\section{Methods and Materials}

\section{Imaging System}

All patients were imaged with a multi-wavelength frequency domain instrumentation system [documented earlier (32)] which collects amplitude and phase measurements of light reflectance, after passing signals through the pendant breast at five or six wavelengths in the near infra-red range. The wavelengths were specifically, $661,761,785,808,826$, and $849 \mathrm{~nm}$ and the use of five wavelengths excluded the availability of $849 \mathrm{~nm}$. The imager is designed for a non-compressive, cross-sectional breast exam (using data from three planes spaced $1 \mathrm{~cm}$ apart) involving 240 measurement points in each slice at each wavelength acquired with a configura- 
tion of 16 sources and 15 detectors. The measurements are calibrated to compensate for system offsets and an initial estimate close to the bulk background tissue properties is obtained from a homogeneous calculation of the diffusion equation for the relevant breast size.

A finite-element model to the diffusion approximation for the radiative transfer equation, is used for image reconstruction to simulate the way that photons diffuse through tissue due to the multiple scattering processes that occur as they travel $(33,34)$. Studies have shown that this model allows reasonable separation of the absorption and scattering processes of light within tissue (35) and has been utilized in reconstruction algorithms to produce images of absorption and reduced scattering coefficients $\left(\mu_{a}, \mu_{s}{ }^{\prime}\right.$, respectively). A Newton-Raphson iterative scheme for minimization of measured and model-calculated data, along with Levenberg Marquardt regularization have been utilized to obtain these images. This followed by spectral fitting has been termed as the "conventional" method of image recovery, in this work. This model for image reconstruction was recently extended to incorporate spectral a priori information related to the behavior of the absorbers and scatterers as a function of wavelength (9). Assuming the main absorbers in the tissue are oxy-hemoglobin $\left(\mathrm{HbO}_{2}\right)$, de-oxyhemoglobin $(\mathrm{Hb})$ and water (within this wavelength band) and knowing their molar absorption spectra (absorption per unit concentration) at the six wavelengths, it is possible to calculate each of their contributions to the absorption using Beer's law:

$$
\mu_{a}(\lambda)=\sum_{i=1}^{3} \varepsilon_{i}(\lambda) c_{i}
$$

where $\varepsilon_{i}$ is the molar absorption spectra of the $i^{\text {th }}$ chromophore at wavelength $\lambda$ and $c_{i}$ is the chromophore's concentration. In this way, images of these chromophores as well as extended indices given by $\mathrm{Hb}_{\mathrm{T}}=\mathrm{HbO}_{2}+\mathrm{Hb}$ (in $\mu \mathrm{M}$ ), and oxygen saturation as $\mathrm{S}_{\mathrm{t}} \mathrm{O}_{2}=\mathrm{HbO}_{2} / \mathrm{Hb}_{\mathrm{T}} \times 100$ (in $\%$ ) can be obtained from the absorption images.

An empirical approximation to scattering behavior predicted by Mie theory for particles approaching sizes larger than the wavelength was introduced by Van Steveran (36) and Mourant et al. (37) and has been in several studies to derive associated parameters. The relationship is given by:

$$
\mu^{\prime}(\lambda)=a \lambda^{-b}
$$

This fit can be used to estimate scatter amplitude $(a)$ and power $(b)$ images with wavelength in $\mu \mathrm{m}$, based on $\mu_{s}^{\prime}$ images at the six wavelengths obtained from the image reconstruction. The coefficient $\mu^{\prime}$ has units $\mathrm{mm}^{-1}$ and $b$ is dimensionless so that $a$ has units given by $10^{-3 b}(\mathrm{~mm})^{b-1}$. Scattering power is governed by the shape or slope of scat- tering which is predominantly affected by the size distribution of membrane bound scatterers within tissue $(38,39)$; the amplitude relates to the number density of these scatterers. Together, these two parameters may reflect variations in breast structural composition due to different sub-cellular organelle and ultra-structural sizes/densities within the fatty, fibroglandular, collagen, and epithelial tissue compartments.

Relationships from Equations [1] and [2] form the basis of the "spectral approach" to image reconstruction: this involves direct recovery of images of the concentrations of $\mathrm{HbO}_{2}, \mathrm{Hb}$, water, scatter amplitude, and power by coupling multi-wavelength measurements together. This approach also uses the Newton's method along with the LevenbergMarquardt regularization, but now the minimization includes all wavelengths' measurements. The technique reduces the total number of unknown parameters in the image reconstruction (from number of wavelengths times optical properties to overall five parameters) and makes the inverse problem better posed by increasing the stability to noise in the measured data. The initial guesses for the functional parameters are set using a constrained least squares fit on the initial optical properties; the optical properties were in turn obtained using a homogeneous fitting algorithm where data was averaged over all source positions and the assumption of a homogeneous medium was used on the circular mesh. Details can be found elsewhere (23). Optimized in terms of obtaining initial estimates of the parameters, regularization, convergence criteria, filtering, and to allow for best calibration procedure for data; and validated in homogeneous imaging fields and simulations $(9,25)$, this method is further evaluated here in experimental phantom measurements where the results indicate that higher qualitative and quantitative accuracy as well as reduced cross-talk between the functional parameters is achieved.

\section{Human Subjects}

As part of a clinical trial to investigate alternate imaging modalities for diagnosis of breast cancer, NIR imaging was carried out on women with mammographically-detected abnormalities. All clinical work was approved by the institutional committee for the protection of human subjects at Dartmouth, and informed consent was obtained from all participants. The women were imaged in the prototype NIR tomography system at the Dartmouth Hitchcock Medical Center (DHMC). The mammograms were interpreted by a radiologist specializing in breast imaging (S.P.P.). The clinical information utilized in the study included the radiographic density, the location of the tumor and its size, in addition to architectural features. The breast radio-density was categorized according to the BI-RADS system (40) as classifications of (i) almost entirely fat, (ii) scattered fibroglandular tissue, (iii) heterogeneously dense, or (iv) extreme- 
ly dense. Pathologic studies were completed following biopsy (WAW) to provide tumor histologic type, grade, and size. In addition, using reproducible, computer-assisted image processing methodology described elsewhere (41), the mean vessel density of the tumor and the ratio of malignant epithelium to surrounding stroma (epithelial to stroma ratio) were also assessed. The results from six women with breast tumors, three of which were diagnosed as infiltrating ductal carcinomas, one with fibrosis, and two with fibrocystic disease; were analyzed here using the spectrally constrained direct chromophore and scattering reconstruction. The contrast between the tumor and background was documented along with interpretation of the images and their correlation to mammography, pathology, and the physiology of the different types of cancers.

\section{Results}

Application to Heterogeneous Experimental Measurements: Phantom Study

In order to test the spectrally-constrained image reconstruction approach on experimental measurements in a heterogeneous geometry, a cylindrical phantom consisting of gelatin with whole blood added for absorption and Titanium dioxide for scatter was made. Two such gelatin phantoms with a diameter of $8.2 \mathrm{~cm}$ were obtained from the same mixture, one of which was maintained in its homogeneous state, and the other had a $2.5 \mathrm{~cm}$ cylindrical hole drilled to have its boundary $1 \mathrm{~cm}$ from the external boundary. This hole was filled with a saline solution containing $4 \%$ pig blood (the hematocrit level of the blood was measured by a clinical co-oximeter so that $4 \%$ blood $=43.2 \mu \mathrm{M}$ total hemoglobin) with $0.75 \%$ Intralipid for scattering. The background chromophore concentrations and scatter for the gel were determined by imaging the phantom in its homogeneous state and using the mean from the reconstructed NIR images, ignoring contributions close to the boundary (taken empirically to be
$12 \%$ of the total diameter); earlier experiments have shown that concentrations obtained this way are quantitatively accurate (9). The inclusion had a contrast of nearly $2: 1$ in total hemoglobin and was expected to have $100 \%$ oxygen saturation and water content. The scattering images were expected to be almost homogeneous because $0.75 \%$ Intralipid was measured to be similar in scattering quantitatively to the background gelatin in the phantom. Amplitude and phase data were collected on this heterogeneous phantom at the six wavelengths and image reconstruction was carried out using both the conventional technique of separate wavelength reconstruction as well as the spectrally-constrained procedure. The top row in Figure 1 shows true images for the five NIR parameters, followed by the images generated with the spectrally-constrained reconstruction and the images from the conventional technique (in the bottom-most row).

The images obtained with the spectrally constrained reconstruction are qualitatively much smoother and more accurate than their conventional counterparts. The simultaneous use of six wavelengths of data along with the spectral priors makes the inverse problem better posed and this along with the parameter reduction procedure provides the smoothness in the spectral images. Images from the conventional technique have more spatial artifacts, including higher cross-talk between oxy-hemoglobin and water, which have similar spectral behavior, resulting in under-estimation of total hemoglobin in the anomaly and saturation of water. In order to obtain the mean, peak, and standard deviation in the anomaly, a FWHM regionization was performed on the images, using the total hemoglobin image for the spectral method and the oxygen saturation image for the conventional technique. While the peak in total hemoglobin still reaches $98 \%$ of the true value, the mean in the anomaly is lower (80.6\%) with significant standard deviation $(\sim 10 \%$ of the mean). The scatter images from the conventional method suffer from a ring-type artifact, which arises from the difficulty in separating the two scatter parameters. This results in

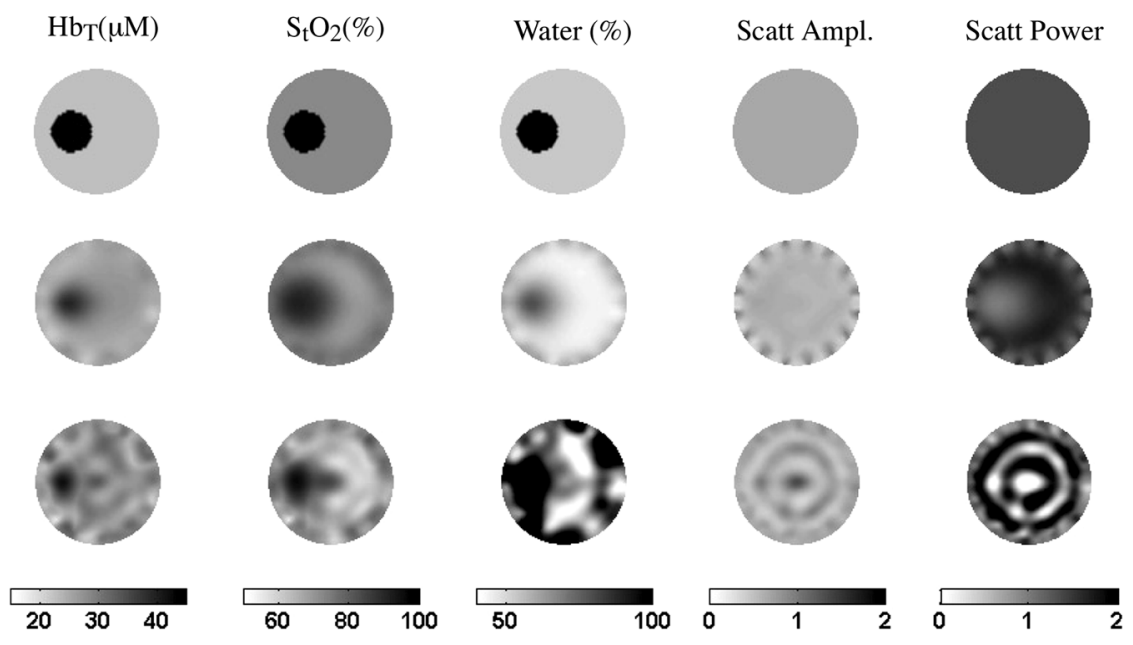

Figure 1: Comparison of images obtained with the spectrally constrained direct chromophore reconstruction and the conventional technique of independent optical property at separate wavelengths recovery on measurements from a gelatin phantom $(8.2 \mathrm{~cm}$ total size) with a $2.5 \mathrm{~cm}$ inclusion. The gelatin phantom contained whole blood and $\mathrm{TiO}_{2}$ for scattering and the inclusion was filled with $4 \%$ pig blood and $0.75 \%$ Intralipid in buffered saline. The expected images are shown in the top row for $\mathrm{Hb}_{\mathrm{T}}(\mu \mathrm{M})$, oxygen saturation $(\%)$, water $(\%)$, scatter amplitude, and scatter power. The spectral method images appear in the second row while conventional technique images are located in the bottom-most row. 
high standard deviation in the images, close to $25 \%$ of the mean for scatter amplitude and $82 \%$ of the mean for scatter power in the anomaly region. This has been circumvented in the spectrally constrained reconstruction through prior information related to scattering, providing well-defined limits for scatter amplitude and power. The standard deviation is now reduced to $2.3 \%$ of the mean for scatter amplitude and $7 \%$ for scatter power. The mean of the scatter amplitude in the anomaly region is quantitatively accurate with a mean error of $3.3 \%$ (reduced from $7 \%$ for the conventional method); and similarly for scatter power, the mean error is reduced to $19 \%$ from $32.4 \%$ for the conventional technique.

The spectral shapes of oxy-hemoglobin and water embedded into this reconstruction minimizes the crosstalk between these parameters and provides accuracy in total hemoglobin in the anomaly within $91.6 \%$ of expected value for the peak and within $84 \%$ for the average and standard deviation reduced by almost half, to $4.83 \%$ of the mean. Water content has been underestimated by $20.3 \%$ using the spectral method. This may be due to the steep difference in background and anomaly water content (typically not observed in a patient), making it difficult to recover the high contrast in the anomaly. A possible solution to improving the accuracy of water content is the use of data in the longer wavelength range $(850-1000 \mathrm{~nm})$. This strategy has been examined in simulations (not shown here), which will incorporate stronger features of water.

\section{Variation of Hemoglobin and Scattering}

In order to investigate the response of the spectrally-constrained algorithm to changes in total hemoglobin, the inclusion's blood concentration was varied systematically from 20 to $44 \mu \mathrm{M}$ and measurements at six wavelengths were taken. The resulting images for total hemoglobin recovered using the spectral priors are shown in Figure 2, along with a comparison to the images obtained from the conventional technique and the expected images.

The images presented in Figure 2 are consistent with those reported from previous experiments and simulations. Specifically, the spectral reconstructions now not only appear smoother but also follow the variation in $\mathrm{Hb}_{\mathrm{T}}$ more accurately than the conventional method without the use of priors. The mean and standard deviation for all NIR parameters in the region of the inclusion were obtained using a FWHM segmentation procedure on the total hemoglobin image. In this calculation, the anomaly was defined as the region containing hemoglobin values above a threshold (midway between the maximum and average of the values for the image). The mean in the anomaly recovered from the spectral method tracked the change in blood concentration with an average error of $12.3 \%$. The other parameters remained constant through this change with a lower standard deviation for the spectral method $(<5 \%$ overall compared to $23.5 \%$ for the conventional method, as percent of the mean).

Imaging the effect of scattering was similarly examined by varying the Intralipid concentration in the $25 \mathrm{~mm}$ inclusion in the gelatin phantom from $0.75 \%$ to $1.75 \%$ in steps of $0.25 \%$; while keeping the total hemoglobin concentration fixed. This change results in an increase in the number density of scatterers while their size remains the same. The Mie theory approximation to scattering behavior in the regime where scatterer size is comparable to the wavelength, predicts that the number density only changes the magnitude of the scattering coefficients, but not the spectral shape or scatter power parmeter (38). This means that an increase in Intralipid concentration translates to a change in scatter amplitude with scatter power remaining the same. The images for scatter amplitude following the change in Intralipid percentage are shown in Figure 3 for the spectrally constrained procedure. Images obtained from the conventional method and the

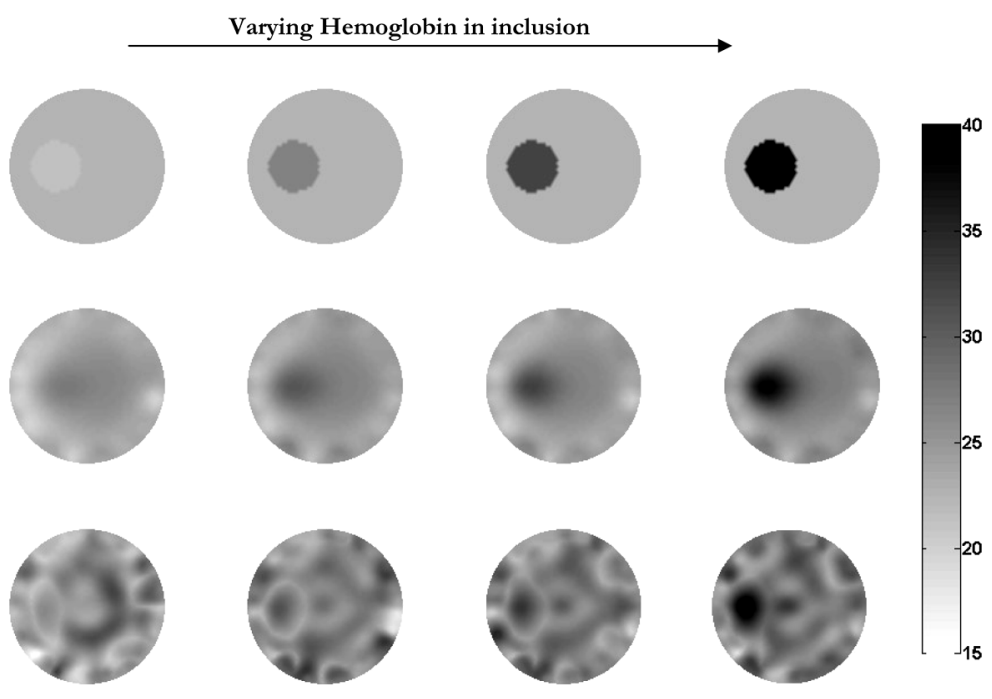

Figure 2: Images of a gelatin phantom whose inclusion $\mathrm{Hb}_{\mathrm{T}}$ concentration was varied from $20-44 \mu \mathrm{M}$. Top row shows true images for $\mathrm{Hb}_{\mathrm{T}}$. Images obtained by application of spectral priors appear in second row while the bottom row contains the images resulting from the conventional technique. The images for all other parameters (oxygen saturation, water and scatter) remained nearly constant with the $\mathrm{Hb}_{\mathrm{T}}$ change. 


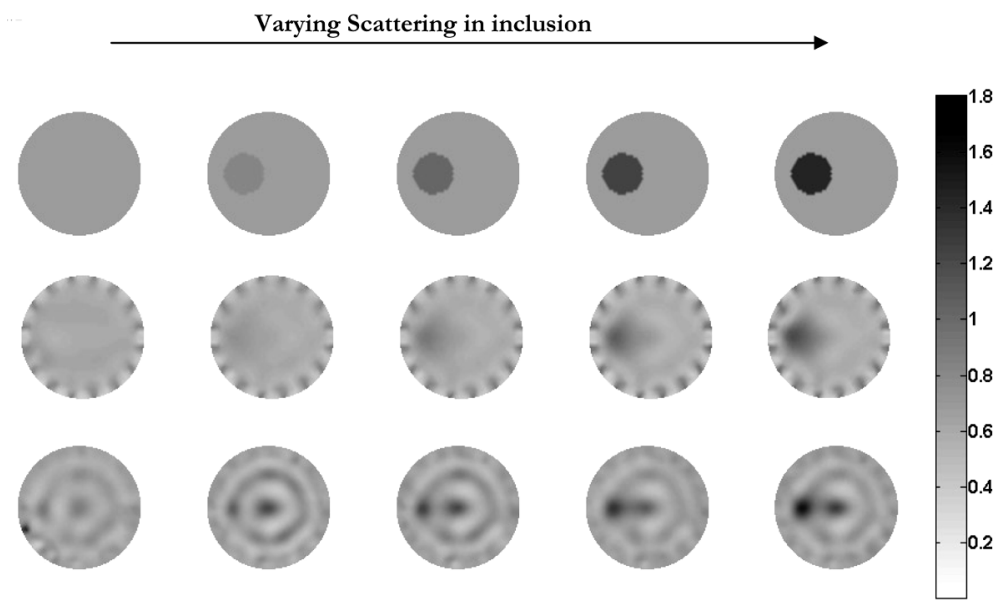

Figure 3: Images of a gelatin phantom whose inclusion Intralipid concentration was varied from 0.75 to $1.75 \%$ in steps of $0.25 \%$. Top row shows true images for scatter amplitude (in units of $10^{-3 b}(\mathrm{~mm})^{b-1}, b$ being scatter power). Results obtained by application of spectral priors appear in the second row while the bottom row contains the images generated by the conventional technique. The images for all other parameters $\left(\mathrm{Hb}_{\mathrm{T}}\right.$, oxygen saturation, water, and scatter) remained relatively constant with scattering changes. expected images are also included. The expected values were obtained from the homogeneous experimental results.

While the images for both the spectral and conventional methods in Figure 3 reveal a localized increase in scatter amplitude in the region of the anomaly, the conventional method generates a ring type artifact found in Figure 1, potentially due to coupling with scatter power. This makes it difficult to discern the inclusion in the conventional images for the first three contrast changes in the experiment. The effect is eliminated in the spectrally reconstructed images, which exhibit a smoother variation with change in scattering. Artifacts close to the boundary exist in the spectral scattering images and this may illustrate the need for increased regularization compared to the other parameters in the direct reconstruction. Scatter amplitude reconstructed using the direct spectrally constrained procedure was found to follow the variation in Intralipid percentage successfully with a mean error of $17.6 \%$. Here, the error increased from $3 \%$ at the lower Intralipid percent to $27.5 \%$ at higher Intralipid concentrations due to scattering. Scatter power stayed constant as expected, with a mean value of $1.19 \pm 0.16$ for the conventional reconstruction procedure and $1.23 \pm 0.03$ from the spectral method, both of which are comparable to the expected value of 1.4 from van Staveren et al. (36). The total hemoglobin, oxygen saturation and water content remained approximately constant with scattering variation, as expected.

\section{Imaging of Infiltrating Ductal Carcinomas (IDCs) (Case Studies 1-3)}

Case Study 1: NIR tomographic images were obtained from a 73-year-old female volunteer (patient ID \#1035) with breast cancer. The left craniocaudal and magnified close-up mediolateral views (22) of the mammogram are shown in Figures 4(a) and (b). They revealed a peripherally located spiculated mass of size $2.5 \mathrm{~cm}$ (maximum focal diameter) at 2:30 in clock face position with architectural distortion occurring over a much larger area (up to $6 \mathrm{~cm}$ ). The patient also went through ultrasonography which confirmed the existence of a hypoechoic irregular mass in the same location. Multiple simple cysts were also evident. Following a $1.1 \mathrm{~cm}$ core needle biopsy, the tumor was diagnosed as infiltrating ductal carcinoma with lobular features. The subject underwent the NIR clinical exam two weeks after the biopsy; the images obtained by applying spectrally constrained reconstruction, along with the conventional method of recovering the optical properties at separate wavelengths, are shown in Figure 4(c). The mid-plane (marked to contain the tumor) measurements at five wavelengths, excluding $849 \mathrm{~nm}$, were used and the breast diameter was $10.4 \mathrm{~cm}$ corresponding to this plane. The breast density was categorized as heterogeneously dense (category 3 ) and pathological examination of the tumor through the biopsy sample revealed a vessel density of $0.9 \%$.

The total hemoglobin image obtained from the spectral reconstruction shows a localized increase at the site of the tumor along with a reduction in oxygen saturation. The oxygenation decrease, an indication of an hypoxic situation in the tissue, is not observable in the images from the conventional method. This finding is consistent with results generated from simulated data (with $1 \%$ random Gaussian noise) (25) and other experiments which showed that the spectral method is quantitatively and qualitatively superior when tracking a change in oxygenation. Imaging of the tumor oxygenation response is a strong indicator of the potential of NIR tomography to study hypoxic fractions in malignancies. The increase in hemoglobin is consistent with earlier studies (2) and the theory that angiogenic activity results in increased vascularity $(29,30)$. The conventional method shows higher values for total hemoglobin in the tumor, compared to the spectral method, with the water content saturating in most of the image, at $100 \%$. Corlu et al. $(9,10)$ have also reported this trend in a carcinoma, indicating a false-increase in hemoglobin with the conventional technique, possibly due to a cross-talk between oxy-hemoglobin and water. The water image using the conventional method 
shows significant artifacts; however, the artifacts are removed in the spectral method in which case water shows a clear increase at the location of the tumor. This is consistent with the expected physiological changes in a tumor which include a leaky vasculature formed in the process of angiogenesis that is responsible for creating an interstitium containing inflammatory cells, fibroblasts, and other enzymes facilitating cell growth (29).

The scatter images from the conventional method are more artifacts-ridden with abnormally high values for amplitude at certain locations as well as negative values for power, that do not correspond to the scattering model predictions. However, with the spectral approach, both scatter amplitude and power show a localized increase at the site of the tumor. Scatter amplitude from the Mie theory approximation, is found to be more sensitive to the number density of the scatterers whereas their size is usually governed by the slope of scattering or the scatter power (38). Hence, we expect the change in scatter amplitude due to cell proliferation to be more localized than scatter power. The contrast in scatter power is possibly due to fibrosis occurring in malignancies which explains the increased optical density.
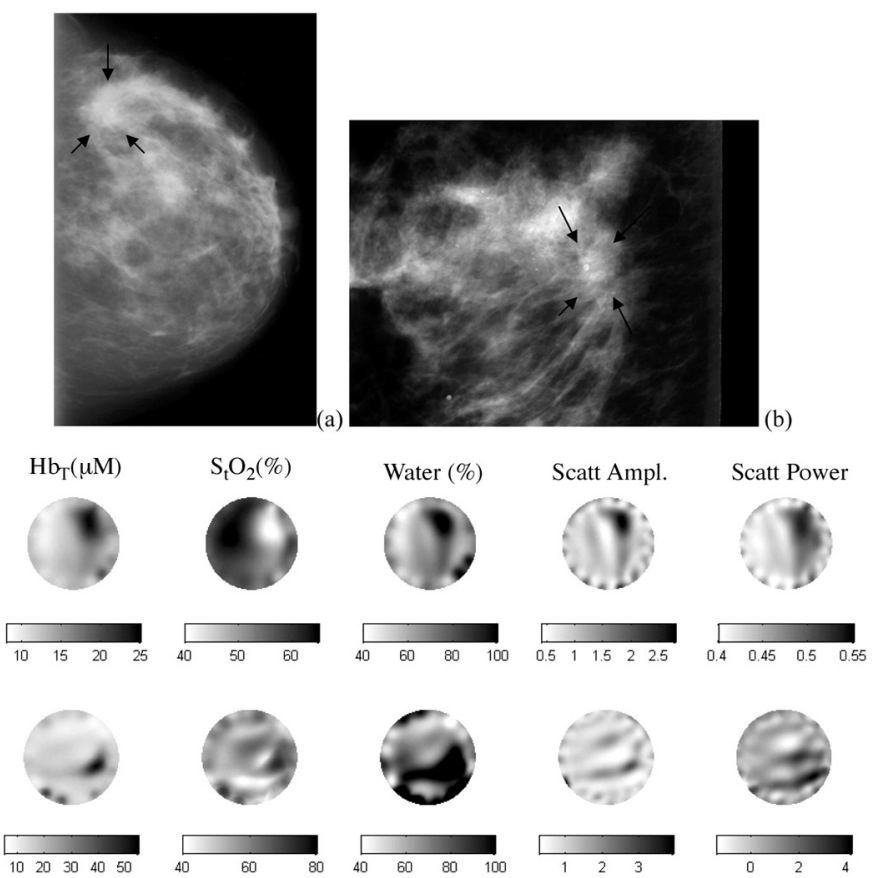

(c)

Figure 4: (a) left craniocaudal mammogram and (b) magnified close-up mediolateral mammogram (22) from the left breast of a 73-yr-old woman with a $2.5 \mathrm{~cm}$ spiculated mass (marked with black arrows) diagnosed as infiltrating ductal carcinoma by core-needle biopsy. (c) top row shows images for $\mathrm{Hb}_{\mathrm{T}}(\mu \mathrm{M}), \mathrm{S}_{\mathrm{t}} \mathrm{O}_{2}(\%)$, water $(\%)$, scatter amplitude, and scatter power obtained using the spectrally constrained direct chromophore reconstruction; bottom row shows the images of the same using the conventional separate wavelength approach. Images in top row correspond well with tumor location (given as 2:30 clock face position, close to the periphery) from the mammogram.
It is evident that the images from the spectrally constrained reconstruction show physiological changes more clearly, with undeniable improvement in image quality. The accuracy of the method has already been validated and henceforth only the spectral method is used to analyze breast cancer images presented. Based on the five NIR parameter images from the top row in Figure 4(c), the average in the tumor and the background were obtained from a FWHM criterion applied to the total hemoglobin image. This ensures that the tumor size, while close to the true size, is specific to the NIR imaging modality and defined accordingly. The criteria has been applied successfully in earlier studies (42). Figure 5 shows the results when the approach is applied to the images above from the spectrally constrained reconstruction. The total hemoglobin increased to a maximum of $24.5 \mu \mathrm{M}$ in the tumor, while the background had a mean of $13.2 \mu \mathrm{M}$, resulting in an increase by $86 \%$ in tumor versus surrounding tissue. A decrease in oxygen saturation was observed at the location of the tumor reducing by nearly $13 \%$ from an average of $56.4 \%$ in the background to $43.6 \%$ in the tumor. Water showed an average increase of up to $89 \%$ in the cancer relative to $65 \%$ in the background, which may also be due to the existence of the cysts found in the sonogram. The tumor also showed a contrast of nearly twice that of scatter amplitude (mean of 0.98 in the background to 2.0 in the tumor). The contrast in scatter power was less pronounced, though an increase in tissue density at the cancer location could still be observed.

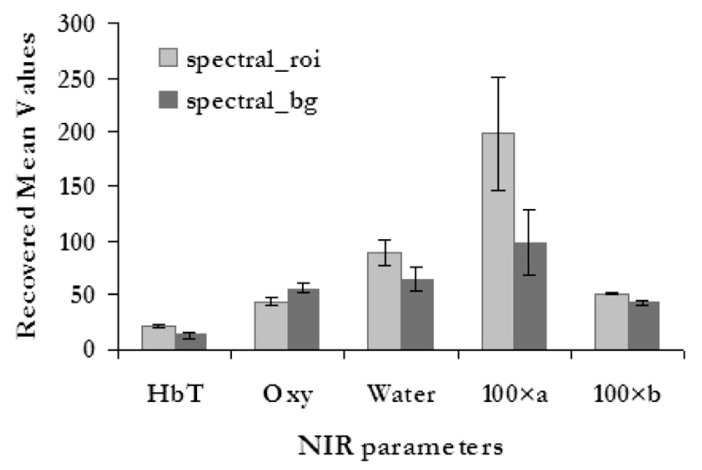

Figure 5: The average for the five NIR parameters along with standard deviation in the region of the tumor and the background (for the patient corresponding to Figure 4). The tumor was defined by segmentation of the images obtained from the spectrally constrained direct chromophore reconstruction using the FWHM criterion. The tumor exhibits increases in total hemoglobin $\left(\mathrm{Hb}_{\mathrm{T}}\right)$, water, and scatter with decreases in oxygenation, compared to the surrounding tissue.

Case Study 2: In the second case, the NIR clinical examination was carried out on a 37-year-old female volunteer (patient ID\# 1077) to image a palpable mass. Mammography revealed a $3 \mathrm{~cm}$ irregular lesion centrally located at the $6: 30$ clock face position, with ill-defined margins in both the left craniocaudal and left mediolateral oblique mammograms [left craniocaudal mammogram and magnified view are shown in Figures 6(a) and (b)]. The tumor was $5 \mathrm{~cm}$ from 
the chest wall. The patient underwent ultrasonography which showed a solid mass larger than $5 \mathrm{~cm}$ in size [Figure 6(c)]. The tumor was confirmed by a biopsy to be an infiltrating ductal carcinoma. Pathology studies on the resected specimen indicated a vessel density of $0.74 \%$. The radiographic classification of the breast was heterogeneously dense. The amplitude and phase data were obtained from the periphery of both breasts and the measurements at five wavelengths (excluding $849 \mathrm{~nm}$ ) from the tumor-bearing left breast were used by the spectral reconstruction to provide the tomographic images shown in Figure 6(d) for the lowest plane closest to the nipple, where the breast diameter was $8.5 \mathrm{~cm}$.

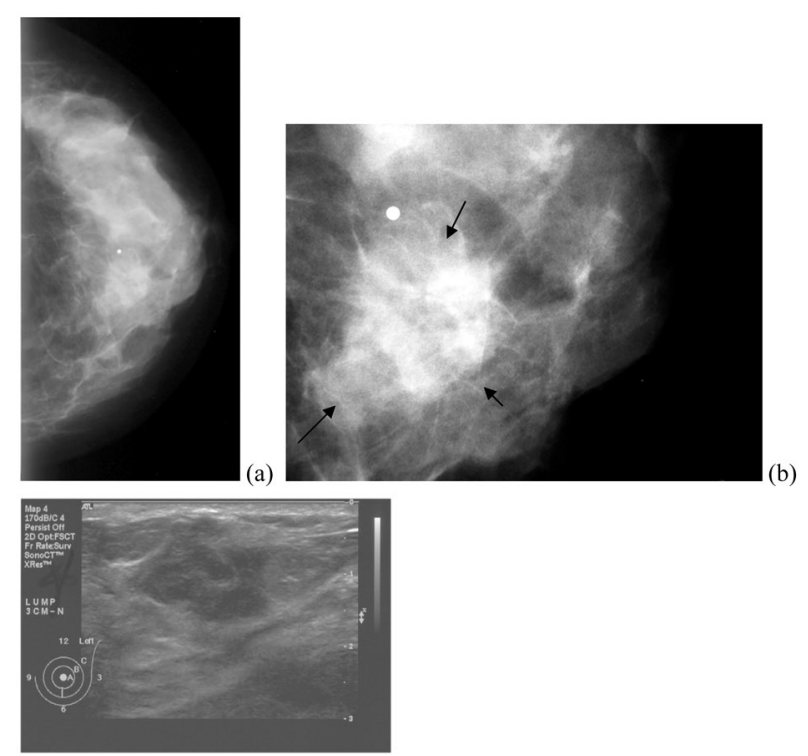

(c)
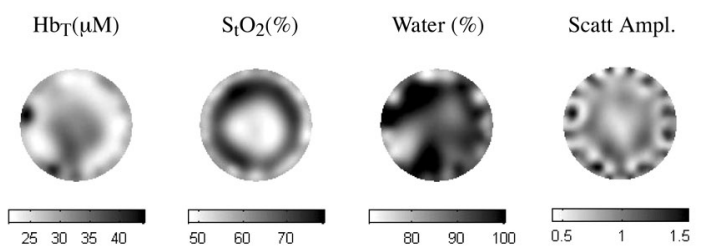

Scatt Power

(d)

Figure 6: (a) left craniocaudal mammogram, (b) magnified view of mammogram, and (c) ultrasound images from the left breast of a 37-yr-old woman with a $3 \mathrm{~cm}$ irregular mass (indicated with black arrows) centrally located at 6:30 on the clock face; diagnosed as infiltrating ductal carcinoma through biopsy. (d) images for $\mathrm{Hb}_{\mathrm{T}}(\mu \mathrm{M}), \mathrm{S}_{\mathrm{t}} \mathrm{O}_{2}(\%)$, water (\%), scatter amplitude, and scatter power obtained using the spectrally constrained direct chromophore reconstruction. The tumor is most clearly visible in the $\mathrm{Hb}_{\mathrm{T}}$ image.

A localized increase in total hemoglobin was found in the location given by the mammogram, increasing to a peak of $37 \mu \mathrm{M}$ in the tumor. Oxygen saturation showed a decrease with respect to the breast background reducing to a minimum of $47.4 \%$, consistent with the prediction of hypoxia in malignancies. A contrast in water was also observed, increasing at the site of the cancer. Scattering did not change appreciably at the cancer site, though the cross-section reveals a mild peak (increasing to 1.1 with respect to a background minimum of 0.5). The lack of a higher contrast in scatter may be due to the fact that the subject was younger in age and had a radiographically dense breast (category 3 ) so that scattering in general is high.

Figure 7 shows the mean and standard deviation for all five reconstructed parameters in the cancer and surrounding tissue following the same FWHM segmentation procedures. Total hemoglobin increased from an average of $27.7 \mu \mathrm{M}$ in the normal tissue to a peak of $37 \mu \mathrm{M}$ (equivalent to an increase by $34 \%$ ) and an average of $34.4 \mu \mathrm{M}$ in the tumor. The oxygen saturation reduced by $6.4 \%$ in the cancer, from an average of $61.7 \%$ in the surrounding tissue. Water increase in the location of the cancer was not as predominant as in the previous case; it changed by less than $5 \%$. This may be due to the large size of the tumor, averaging out the change between the tumor and the surrounding tissue. Scatter amplitude and power remained nearly unchanged in their averages between tumor and the background.

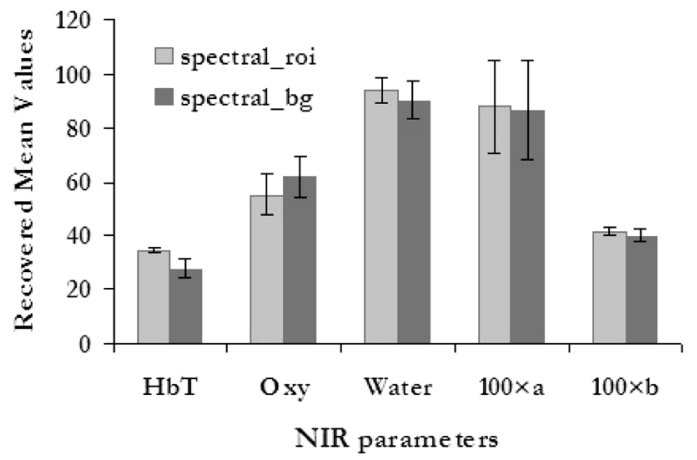

Figure 7: The average and standard deviation for the five NIR parameters in the region of the tumor and the background (for patient corresponding to Figure 6). The tumor was defined by segmentation of the images obtained using the spectrally constrained direct chromophore reconstruction and FWHM criterion. The tumor exhibits an increase in total hemoglobin $\left(\mathrm{Hb}_{\mathrm{T}}\right)$ and water, with decreases in oxygenation, compared to the surrounding tissue.

Case Study 3: In the final cancer case presented here, the subject was a 73-yr-old female volunteer (patient id \# 2034) whose mammogram [see Figure 8(a) and (b)] revealed an approximately $2 \mathrm{~cm}$ focal density with subtle associated architectural distortions in the lower outer left breast at the 4:30 clock position. Just below this was an approximately 1 $\mathrm{cm}$ focal asymmetric density with ill-defined margins, also in the lower, outer left breast. The first abnormality was 6 $\mathrm{cm}$ in depth from the nipple and the second adjacent abnormality was $8 \mathrm{~cm}$ in depth from the nipple. Ultrasound directed to the lower, outer left breast at 4:30, approximately 4.5 $\mathrm{cm}$ from the nipple, demonstrated the two adjacent abnormalities with a questionable hypoechoic bridge between them. The two abnormalities collectively measured $2.5 \mathrm{~cm}$ in greatest diameter. The radio-density of the breast belonged to category 2, the scattered type. Excisional biop- 

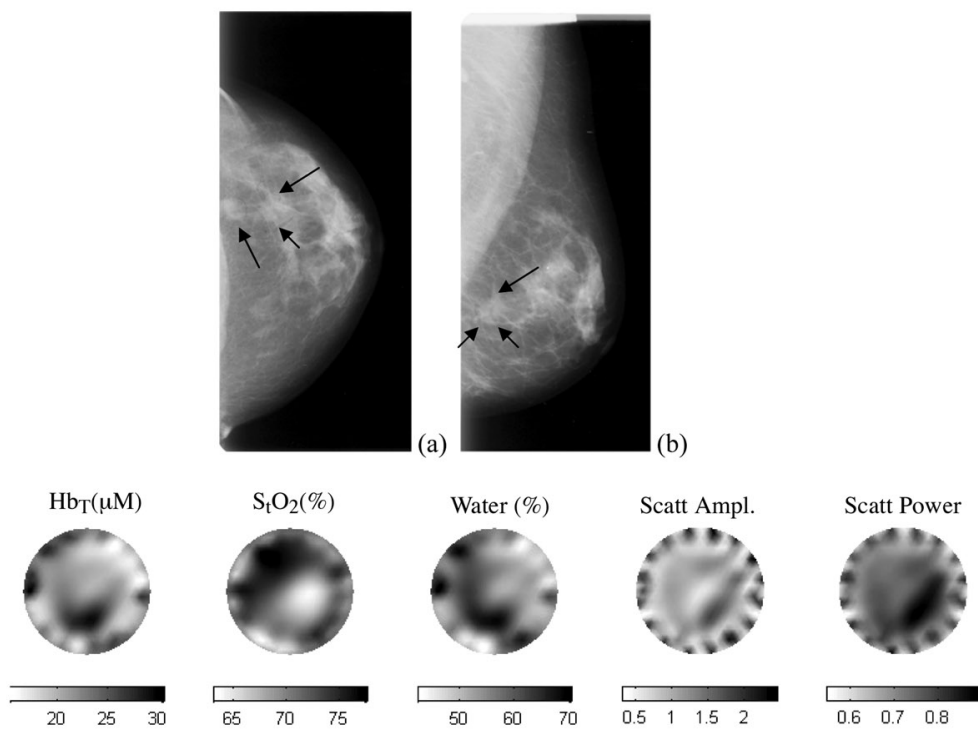

(c)

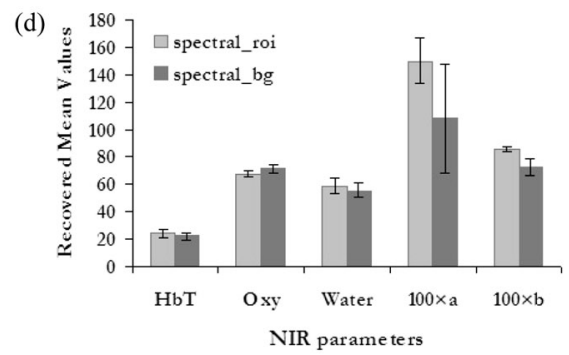

sy confirmed the tumor as an infiltrating ductal carcinoma with a vessel density of 0.71 . On excision, there was a single lesion with a fatty interface and the pathology size of the tumor was $2.5 \mathrm{~cm}$. The NIR exam used six wavelengths and the images obtained, are shown in Figure 8(c) for total hemoglobin, oxygen saturation, water, and scatter.

Total hemoglobin showed an increase at the 6:00 clock position, slightly different from the location of 4:30 given by the Radiologist. Oxygen saturation and scatter show the tumor at the right location, with a reduction in oxygen saturation observed at the cancer site to a minimum of $64 \%$. The water content in the tumor showed an increase with respect to the background with a maximum value of $68 \%$. Both scatter amplitude and power also showed a contrast discerning the cancer from the surrounding tissue.

The cancer observed in the images in Figure 8(c) was regionized using the FWHM approach to zone into the tumor which placed it in the correct location (corresponding to the mammograms). The average and standard deviation was obtained in the lesion and plotted alongside the mean in the surrounding normal tissue in Figure 8(d). The maximum in total hemoglobin in the tumor increased by $36 \%$ relative to the background. Oxygenation and water content did not change substantially but scatter amplitude increased at the location of the cancer by $39 \%$. Scatter power showed a contrast with a value of 0.85 compared to 0.72 in the background.

\section{Human Imaging of a Fibrosis Lesion (Case Study 4)}

Case Study 4: Also studied here are subjects with benign conditions, specifically, fibrosis (a benign, localized lesion comprising an abundance of scar-like, dense stromal collagen in proportion to a reduced epithelial component); and fibrocystic disease. The mammogram of a 49-yr-old female volunteer (patient id \#2135) showed a peripheral tumor in a scattered type breast. The left craniocaudal and mediolateral oblique mammograms (see Figure 9) revealed an approximately $5 \mathrm{~cm}$ area of asymmetric density in the upper outer quadrant of the left breast; the cone compression views of the mammograms gave it the appearance of normal glandular tissue. Ultrasound of the majority of this area showed dense fibroglandular tissue; however, at the 2:30 clock position, $10 \mathrm{~cm}$ from the nipple, was an approximately $2.7 \times 1.7$ $\times 1.2 \mathrm{~cm}$ ill-defined hypoechoic area with dense posterior acoustic shadowing, giving the suspicion of local fibrosis. There were several tiny cysts in the same area. The patient's symptoms and concerning ultrasound lead to a core biopsy which confirmed the lesion as fibrosis, a benign condition, with a vessel density of 0.33 . 


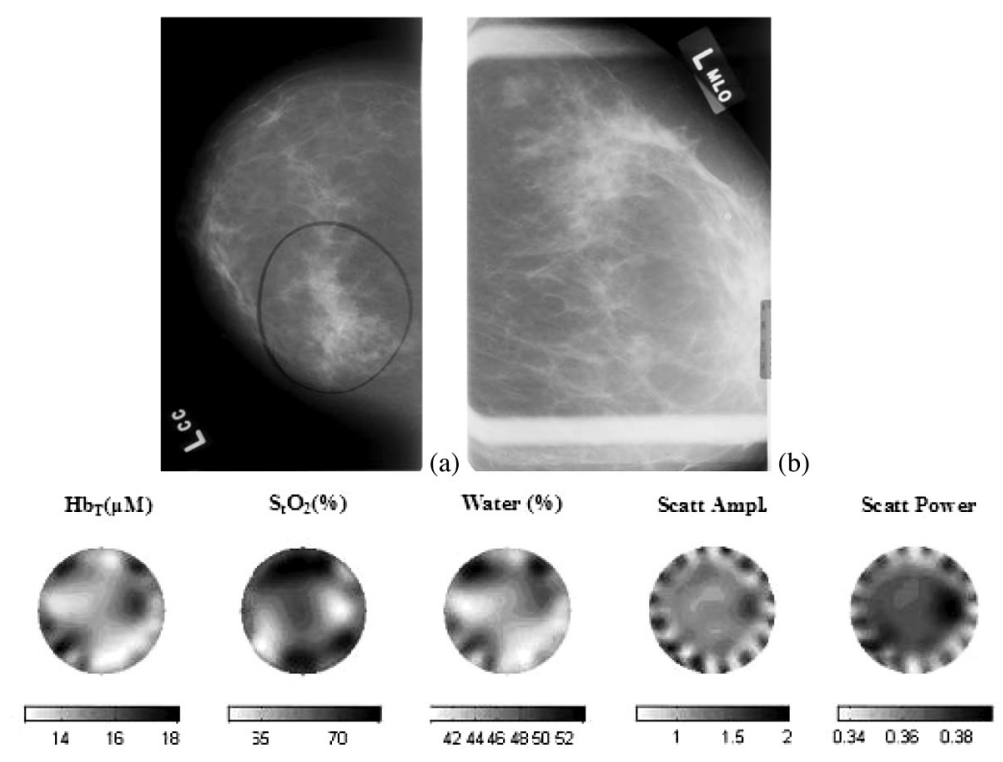

(c)

(d)

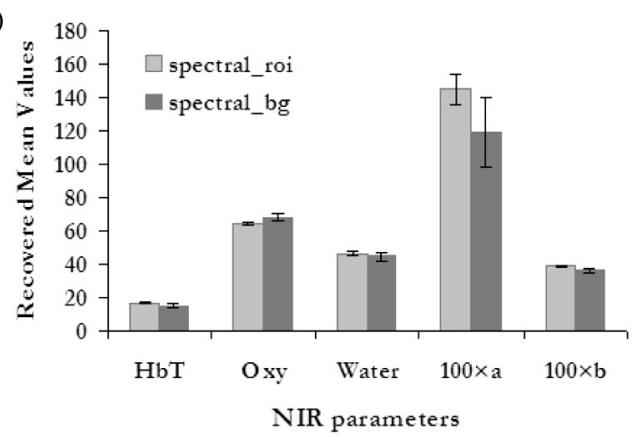

The NIR clinical examination was carried out two days prior to the biopsy. It consisted of collecting three planes of frequency domain data where the measurements from plane 0 (which contained the tumor), closest to the chest wall (at six wavelengths), was used to reconstruct for the NIR parameters. The spectral approach was used and the images are shown in Figure 9(c). The tumor is most clearly visible in total hemoglobin and scatter amplitude and power. Total hemoglobin showed a slight increase whereas the contrast in scatter is more pronounced. Since the vessel density is much lower compared to the malignancies documented here, the contrast in total hemoglobin is also expected to be low. Fibrosis contains a higher density of tissue which explains the increases observed in the scattering parameters. Scatter amplitude increased to a maximum of 1.6 and a lower contrast is observed in scatter power. Water content exhibited a contrast but overall, has a small range from 42 to $52 \%$ overall. Oxygenation varied by less than $5 \%$ between tumor and background. The tumor and background averages are shown along with standard deviations in Figure 9(d) using the regionization scheme employed in all of the earlier cases. The same trend is visible, with a contrast in the tumor of less than $10 \%$ of the surrounding
Figure 9: (a) Left craniocaudal mammogram, (b) left mediolateral oblique mammogram magnified for a 49-yr-old subject showing a $2.7 \mathrm{~cm}$ mass in the 2:30 clock position, diagnosed by core biopsy as the benign condition, fibrosis. (c) NIR tomographic images obtained by applying the spectral reconstruction on the frequency domain data from the plane closest to the chest wall containing the tumor. The tumor is most clearly discernible in total hemoglobin and scatter in the 3:00 clock position with oxygen saturation and water having almost homogeneous images. (d) The average for the five NIR parameters along with the standard deviation in the region of the tumor and the background for a subject with fibrosis The tumor exhibits a small increase in total hemoglobin $(10 \%)$ and a larger increase in scatter amplitude (22\%) with respect to the surrounding tissue; other NIR parameters do not show much change. tissue in total hemoglobin and scatter amplitude showing the most dominant change, increasing by $22 \%$ in the tumor, from a background value of 1.2.

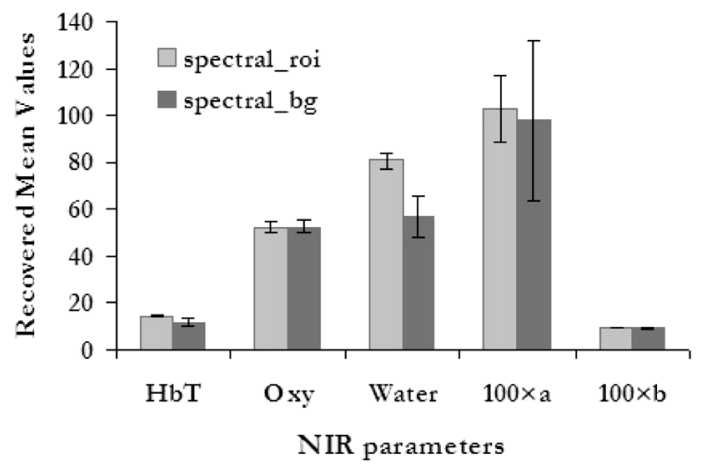

Figure 10: Mean values and standard deviation in the tumor (segmented using FWHM criterion as in previous cases) and surrounding tissue obtained from the NIR images reconstructed with the spectral approach using frequency domain data from the periphery of the breast. The volunteer was a 49-yr-old subject, presenting with an $0.7 \times 0.5 \mathrm{~cm}$ ill-defined mass, diagnosed by stereotactic guided biopsy as the benign condition, FCD. Contrast was observed at the location of the lesion in total hemoglobin and water, but no decrease in oxygen saturation was observed in the images (17). The dominant contrast was found to be in water. 


\section{Human Imaging of fibrocystic disease (FCD) (Case Study 5)}

Case Study 5: Frequency domain measurements were obtained from a 50-year-old female volunteer whose mammogram revealed a $0.7 \times 0.5 \mathrm{~cm}$ ill defined mass in the upper-inner left breast, approximately $9 \mathrm{~cm}$ deep to the nipple. Although portions of the margins were fairly well-circumscribed, at least half was ill-defined or obscured. The lesion was non-calcified and non-palpable and the ultrasound exam of this region showed no sonographic abnormality. A stereotactic guided biopsy revealed the lesion as the benign condition of Fibrocystic Disease (FCD). The NIR parameter images using the spectrally constrained technique applied to frequency domain data at six wavelengths, were discussed in Srinivasan et al. (25). The comparison of the mean in the lesion versus the background is plotted in Figure 10. The dominant contrast was in water, which increased by nearly $24 \%$ from a background content of $57 \%$.

Another female volunteer with FCD imaged with the NIR modality was a 61-yr-old woman (patient id \#1059) with multiple cysts, whose mammogram did not reveal any

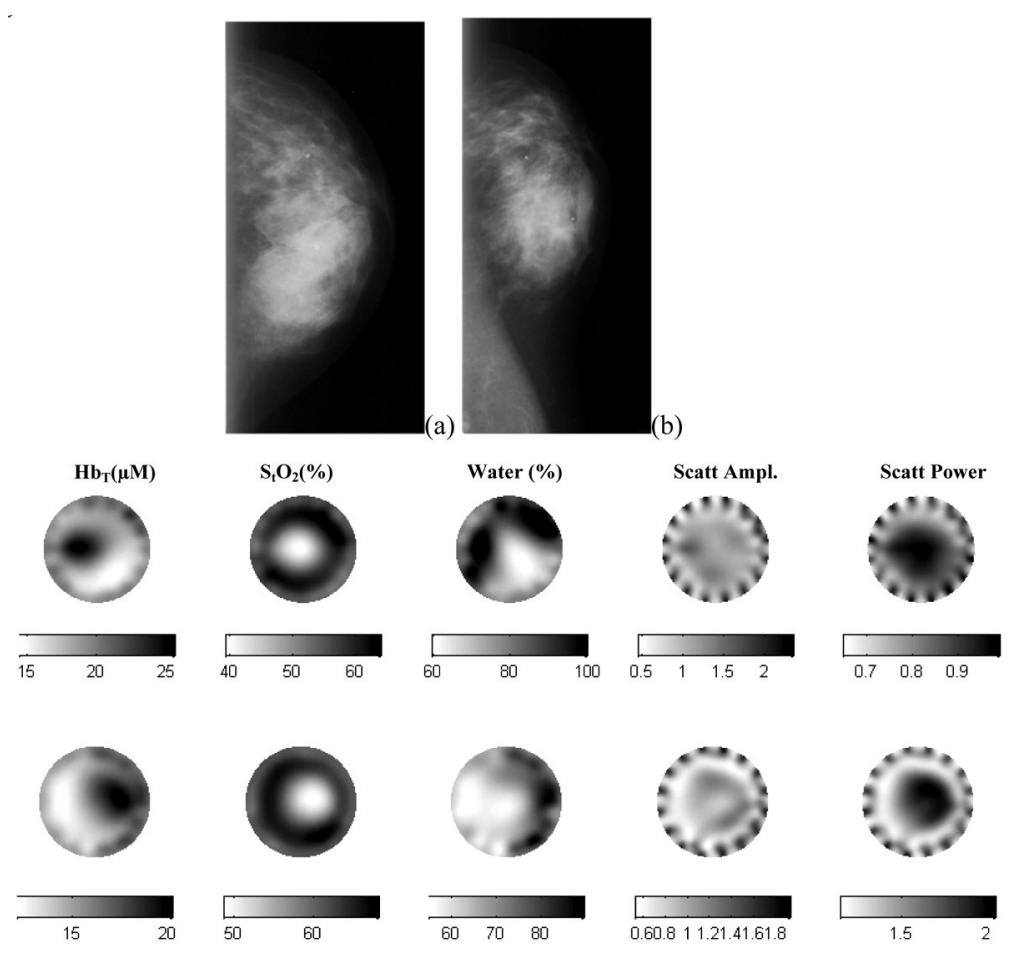

(c)

(d)

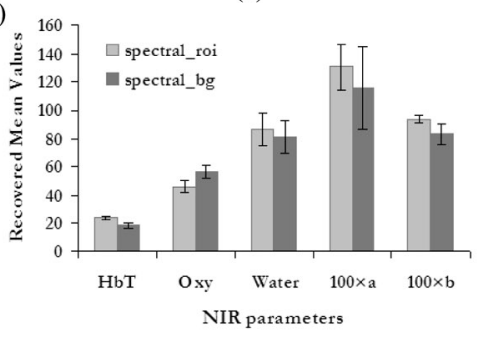

abnormality [see Figure 11 (a) and (b)]. Ultrasound showed the lesion, $1.1 \mathrm{~cm}$ in size, centrally located at 9.30 on the clock face in the right breast and core biopsy confirmed FCD. The subject had a breast density belonging to category 3 (heterogeneously dense), which probably explains why it was difficult to find the cysts, in both the craniocaudal and mediolateral oblique mammograms. The volunteer was taken through the NIR clinical exam and all six wavelengths were used for the tissue interrogation.

The images are shown in Figure 11(c); the lesion is clearly distinguishable in hemoglobin and scatter as a localized increase in the expected position. There is also a centralized decrease in oxygenation which may confound the diagnosis of this lesion; however, examination of the contralateral breast images [shown in Figure 11(c), bottom row] revealed a similar trend which leads to the conclusion that this change in oxygen saturation is more likely due to the metabolic activity of glandular relative to adipose tissue. Water showed an increase consistent with the previous FCD case study analyzed suggesting that water content is a likely indicator of cysts containing fluids. Scatter amplitude also
Figure 11: (a) Right craniocaudal and (b) right mediolateral

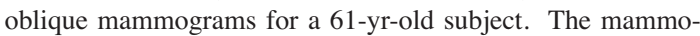
grams showed no abnormality. Ultrasound revealed a $1.1 \mathrm{~cm}$ lesion at the 9:30 clock position diagnosed as FCD. (c) top row shows the images obtained using the spectral reconstruction for the right breast; bottom row shows the images for the contralateral normal breast. Both show a trend of decreasing oxygenation corresponding to an increasing hemoglobin; likely occurring as a result of fibroglandular tissue rather than tumor location. (d) Average and standard deviation in the benign lesion and the surrounding tissue. Water and scatter show a contrast over the background. 
showed a contrast and this maybe an indicator of denser tissue enveloping the cysts. This is also the primary change not visible in the contralateral breast.

\section{Discussion and Conclusions}

Studies have indicated that measurements of neovasculature, hypoxia and cellular microenvironment could have a role in tumor detection and treatment planning $(13,29)$, as they are all indicators of tissue function, which are important features of solid cancers. NIR tomography is an imaging method which can directly quantify some of these functional processes with non-invasive imaging. Hemoglobin should be a measure of the vascularization of tissue; oxygen saturation offers an indirect measure of tissue oxygenation which relates to partial pressure of oxygen in tissue, and hence to a direct measure of hypoxic fractions in tumors. Water is a measure of the microenvironment and cellular compartments (26) and scattering is related to subcellular organelle density. These together provide noninvasive measures through which to study changes in the breast from malignanices and benign lesions.

While correlation plots of NIR cancer and benign clinical data are useful to identify overall trends separating malignant from benign disease, [an example is the hemoglobinoxygen saturation plot shown in Grosenick et al. (24)] the reality may be that separating the two is more complicated. Certain features of benign conditions, such as fibrocystic disease, present difficulties in diagnosing it from cancer, when based only upon mammographic image features (30). NIR tomography is undergoing several clinical trials evaluating its role in aiding breast lesion diagnosis $(2,18)$; however, defining the particular NIR characteristics expected in different types of tumors is not yet well defined. While NIR gives estimates of oxygenation in the tumor and surrounding tissue, hypoxia is not a sole indicator in tumor diagnosis, as Okunieff et al. (13) showed that some malignancies may not have $\mathrm{pO}_{2}$ values less than $5 \mathrm{~mm} \mathrm{Hg}$. In the three case studies presented here for infiltrating ductal carcinomas each had reduction in oxygen saturation. However, the change varied from a decrease of $4 \%$ to $13 \%$ from the surrounding oxygenation, indicating that if this decrease is accurate, the contrast available is likely subtle. A similar decrease was also observed in the FCD case study (patient id \#1059), where the reduction was more likely due to the higher metabolism of fibroglandular tissue; this was verified by a similar trend of a central lower oxygenation in the normal contralateral breast, corresponding to both a more vascularized tissue region as well as an increase in scatter power indicative of higher radio-density. While more clinical cases will be required to validate the trend shown here, it may be that oxygen saturation alone is not sufficient to separate cancer from FCD. Hence, it is likely more useful to examine the charac- teristics of different tumors in the NIR images along with the comprehensive data sets which are available from mammograms, ultrasound and potentially MR images.

The NIR images presented here appear to be the most accurate representation of these functional quantities possible, due to the use of the direct spectral reconstruction algorithm. The spectrally-constrained image reconstruction process was validated in phantom experiments and showed a superior ability to follow changes related to total hemoglobin and scattering as well as oxygen saturation [shown before (9)] The images for the case studies presented here are consistent with the physiological changes that would be expected based upon conventional understanding of larger solid tumors. Total hemoglobin showed a localized increase in the malignancies with a higher contrast (34-86\% increase in the lesion with respect to the surrounding tissue) compared to the benign lesions (10-28\% increase in lesion compared to background). Hemoglobin also seemed to correlate with vessel density, obtained through biopsy of tumor tissue, where higher vessel density has been associated with higher hemoglobin content. Using the six cases studied here along with other tumors imaged $(n=12$, seven cancers and five benign lesions, size $>8 \mathrm{~mm}$ ), Figure 12 shows this trend. The data does not include the patient with FCD (patient id \#1059), whose mammogram did not show a lesion; and whose NIR images show an increase in hemoglobin, more likely indicating glandular tissue. The figure supports an interesting observation: typically $1 \%$ human blood in its normal state contains $\sim 20-25 \mu \mathrm{M}$ blood; and $1 \%$ vessel density from the graph shows a correspondence of $1 \%$ blood $=26 \mu \mathrm{M}$. This trend has to be validated in a larger number of clinical cases.

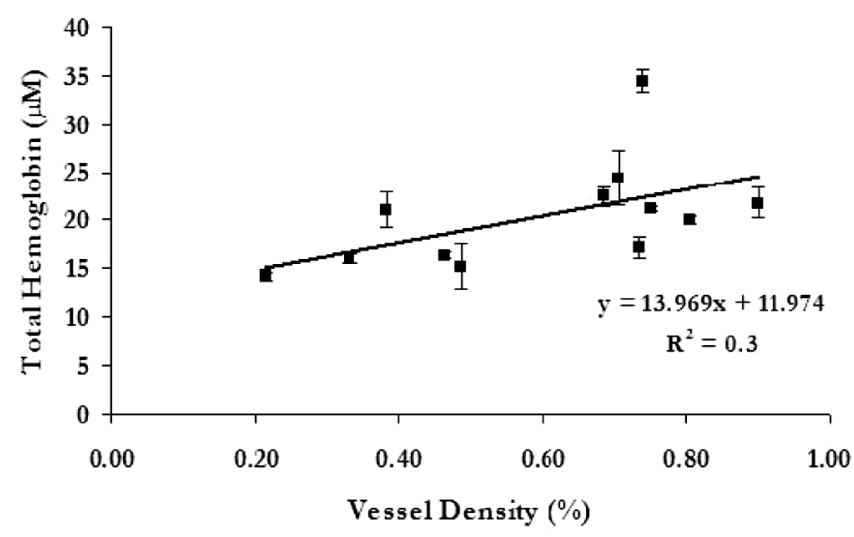

Figure 12: The average total hemoglobin and standard deviation in the location of the lesions from 12 tumors imaged in vivo (seven cancers and five benign lesions) including the case-studies reported in this paper, plotted against vessel density in the tumor obtained from pathology, indicates a possible correlation.

All three carcinomas studied here showed a localized change in all five NIR images; the same was not true in the benign conditions. In the subject with fibrosis, the dominant change 
was in scatter amplitude, consistent with the densely packed fibrous tissue. In cancers, the contrast in scatter amplitude agreed with an expectation of cell proliferation resulting in higher number density of scatterers in the cancers. This yields the encouraging conclusion that scatter amplitude could be an emerging indicator of the density of localized tissues, which when interpreted along with total hemoglobin, oxygenation and water, may enable separation of malignant and benign disease. Scatter power, on the other hand, may be more indicative of bulk radiodensity of the breast $(26,43$, 44). Scattering was found to correlate inversely with $\mathrm{E} / \mathrm{S}$ ratio in preliminary studies and this will be investigated with the spectral approach in future work.

Water content in the images studied here, obtained using the spectrally constrained direct reconstruction, has yielded increased information, compared to the conventional approach of separate wavelength recovery. In all three malignancies, water showed an increase with respect to the surrounding tissue coinciding with the physiological expectation of a leaky endothelium. Water was the dominant mechanism of contrast in the FCDs (see images in Figure 10 and 11) along with scatter change for one of the cases (patient \#1059).

Overall, using the spectrally constrained direct reconstruction approach, the NIR images studied here offer an example of qualitative ways of characterizing different types of lesions. The analysis when performed on a larger number of clinical cases will present distinctive features of tumors useful in diagnosis. The physiological changes observable here can be extended to monitoring response to therapy, predicting risk of malignancy or aggressiveness of tumors. At this stage of development, the role of NIR imaging in diagnosis is still uncertain; however, through algorithm improvement and case study analysis, the potential value of the NIR parameters becomes clearer. It is likely that this system can be used to measure physiological changes which are not observable in other imaging modalities, and further patient imaging sessions will help determine those parameters and indications where the system will have its most important impact.

\section{Acknowledgements}

This work has been supported through NIH grants PO1CA80139 and RO1CA69544 and Department of Defense DAMD17-03-01-0405.

\section{References}

1. B. Chance, Q. Luo, S. Nioka, D. C. Alsop, and J. A. Detre. Optical Investigations of Physiology: A Study of Intrinsic and Extrinsic Biomedical Contrast. Phil. Trans. R. Soc. Lond. B 352, 707-716 (1997).

2. B. W. Pogue, S. P. Poplack, T. O. McBride, W. A. Wells, O. K. S., U. L. Osterberg, and K. D. Paulsen. Quantitative Hemoglobin Tomography with Diffuse Near-Infrared Spectroscopy: Pilot Results in the Breast. Radiology 218, 261-266 (2001).
3. B. J. Tromberg, O. Coquoz, J. B. Fishkin, T. Pham, E. R. Anderson, J. Butler, M. Cahn, J. D. Gross, V. Venugopalan, D. Pham. Non-invasive Measurements of Breast Tissue Optical Properties Using Frequency-domain Photon Migration. Phil. Trans. R. Soc. Lond. B 352, 661-668 (1997).

4. X. Intes, J. Ripoll, Y. Chen, S. Nioka, A. G. Yodh, and B. Chance. In Vivo Continuous-wave Optical Breast Imaging Enhanced with Indocynanine Green. Med. Phys. 30, 1039-1047 (2003).

5. M. A. Franceschini, K. T. Moesta, S. Fantini, G. Gaida, E. Gratton, H. Jess, W. W. Mantulin, M. Seeber, P. M. Schlag, and M. Kaschke. Frequency-domain Techniques Enhance Optical Mammography: Initial Clinical Results. Proc. Nat. Acad. Sci USA 94, 64686473 (1997).

6. X. Gu, Q. Zhang, M. Bartlett, L. Schutz, L. L. Fajardo, and H. Jiang. Differentiation of Cysts from Solid Tumors in the Breast with Diffuse Optical Tomography. Acad. Radiology 11, 53-60 (2004).

7. A. Corlu, T. Durduran, R. Choe, M. Schweiger, E. M. Hillman, S. R. Arridge, and A. G. Yodh. Uniqueness and Wavelength Optimization in Continuous-wave Multispectral Diffuse Optical Tomography. Opt. Lett. 28, 2339-2341 (2003).

8. A. Li, Q. Zhang, J. P. Culver, E. L. Miller, and D. A. Boas. Reconstructing Chromosphere Concentration Images Directly by Continuous-wave Diffuse Optical Tomography. Opt. Lett. 29, 256258 (2004)

9. S. Srinivasan, B. W. Pogue, S. Jiang, H. Dehghani, and K. D. Paulsen. Spectrally Constrained Chromophore and Scattering NIR Tomography Provides Quantitative and Robust Reconstruction. Appl. Opt. 44, 1858-1869 (2005).

10. A. Corlu, R. Choe, T. Durduran, K. Lee, M. Schweiger, S. R. Arridge, E. M. Hillman, and A. G. Yodh. Diffuse Optical Tomography with Spectral Constraints and Wavelength Optimization. Appl. Opt. 44, 2082-2093 (2005).

11. P. Vaupel, K. F., and O. P. Blood Flow, Oxygen and Nutrient Supply, and Metabolic Microenvironment of Human Tumors: A Review. Cancer Research 49, 6449-6465 (1989).

12. M. Hockel and P. Vaupel. Tumor Hypoxia: Definitions and Current Clinical, Biologic, and Molecular Aspects. J. Natl. Cancer Inst. 93, 266-276 (2001).

13. P. Okunieff, M. Hoeckel, E. P. Dunphy, K. Schlenger, C. Knoop, and P. Vaupel. Oxygen Tension Distributions are Sufficient to Explain the Local Response of Human Breast Tumors Treated with Radiation alone. Int. J. Radiation Oncology Biol. Phys. 26, 631-636 (1993).

14. D. M. Brizel, S. P. Scully, J. M. Harrelson, L. J. Layfield, J. M. Bean, L. R. Prosnitz, and M. W. Dewhirst. Tumor Oxygenation Predicts for the Likelihood of Distant Metastases in Human Soft Tissue Sarcoma. Cancer Research 56, 941-943 (1996).

15. P. Vaupel, A. Mayer, S. Briest, and M. Hockel. Oxygenation Gain Factor: A Novel Parameter Characterizing the Association Between Hemoglobin Level and the Oxygenation Status of Breast Cancers. Cancer Research 63, 7634-7637 (2003).

16. A. Bottini, A. Berruti, M. P. Brizzi, A. Bersiga, D. Generali, G. Allevi, S. Aguggini, G. Bolsi, S. Bonardi, G. Bertoli, P. Alquati, and L. Dogliotti. Pretreatment Haemoglobin Levels Significantly Predict the Tumour Response to Primary Chemotherapy in Human Breast Cancer. Br. J. Cancer 89, 977-982 (2003).

17. D. L. Conover, B. M. Fenton, T. H. Foster, and E. L. Hull. An Evaluation of Near Infrared Spectroscopy and Cryospectrophotometry Estimates of Haemoglobin Oxygen Saturation in a Rodent Mammary Tumour Model. Physics in Medicine \& Biology 45, 2685$2700(2000)$.

18. B. J. Tromberg, N. Shah, R. Lanning, A. Cerussi, J. Espinoza, T. Pham, L. Svaasand, and J. Butler. Non-invasive In Vivo Characterization of Breast Tumors Using Photon Migration Spectroscopy. Neoplasia (New York) 2, 26-40 (2000). 
19. D. B. Jakubowski, A. E. Cerussi, F. Bevilacqua, N. Shah, D. Hsiang, J. Butler, and B. J. Tromberg. Monitoring Neoadjuvant Chemotherapy in Breast Cancer using Quantitative Diffuse Optical Spectroscopy: A Case Study. J. Biomed. Opt. 9, 230-238 (2004).

20. V. Chernomordik, D. W. Hattery, D. Grosenick, H. Wabnitz, H. Rinneberg, K. T. Moesta, P. M. Schlag, and A. Gandjbakhche. Quantification of Optical Properties of a Breast Tumor Using Random Walk Theory. Journal of Biomed. Opt. 7, 80-87 (2002).

21. E. Heffer, V. Pera, O. Schutz, H. Siebold, and S. Fantini. Nearinfrared Imaging of the Human Breast: Complementing Hemoglobin Concentration Maps with Oxygenation Images. Journal of Biomed. Opt. 9, 1152-1160 (2004).

22. T. O. McBride, B. W. Pogue, S. Jiang, U. L. Osterberg, K. D. Paulsen, and S. P. Poplack. Multi-spectral Near-infrared Tomography: A Case Study in Compensating for Water and Lipid Content in Hemoglobin Imaging of the Breast. J. Biomed. Opt. 7, $72-79$ (2002).

23. H. Dehghani, B. W. Pogue, S. P. Poplack, and K. D. Paulsen. Multiwavelength Three-dimensional Near-infrared Tomography of the Breast: Initial Simulation, Phantom, and Clinical Results. Applied Optics 42, 135-145 (2003).

24. D. Grosenick, H. Wabnitz, K. T. Moesta, J. Mucke, M. Moller, C. Stroszczynski, J. Stossel, B. Wassermann, P. M. Schlag, and H. Rinneberg. Concentration and Oxygen Saturation of Haemoglobin of 50 Breast Tumours Determined by Time-domain Optical Mammography. Phys. Med. Biol. 49, 1165-1181 (2004).

25. S. Srinivasan, B. W. Pogue, S. Jiang, H. Dehghani, and K. D. Paulsen. Spectrally Constrained NIR Tomography for Breast Imaging: Simulations and Clinical Results. Proc. SPIE 2005 (2005).

26. S. Srinivasan, B. W. Pogue, S. Jiang, H. Dehghani, C. Kogel, S. Soho, J. J. Gibson, T. D. Tosteson, S. P. Poplack, and K. D. Paulsen. Interpreting Hemoglobin and Water Concentration, Oxygen Saturation and Scattering Measured In Vivo by Near-infrared Breast Tomography. PNAS 100, 12349-12354 (2003).

27. M. T. Mandelson, N. Oestreicher, P. L. Porter, D. White, C. A. Finder, S. H. Taplin, and E. White. Breast Density as a Predictor of Mammographic Detection: Comparison of Interval- and Screendetected Cancers. J. Natl. Cancer Inst. 92, 1081-1087 (2000).

28. M. K. Simick, R. Jong, B. Wilson, and L. Lilge. Non-ionizing Nearinfrared Radiation Transillumination Spectroscopy for Breast Tissue Density and Assessment of Breast Cancer Risk. Journal of Biomed. Opt. 9, 794-803 (2004).

29. B. S. Kuszyk, F. M. Corl, F. N. Franano, D. A. Bluemke, L. V. Hofmann, B. J. Fortman, and E. K. Fishman. Tumor Transport Physiology: Implications for Imaging and Image-guided Therapy. AJR 177, 747-753 (2001).

30. S. Thomsen and D. Tatman. Physiological and Pathological Factors of Human Breast Disease that can Influence Optical Diagnosis. Ann. N.Y. Acad. Sci. 838, 171-193 (1998).

31. N. Shah, A. Cerussi, D. Jakubowski, D. Hsiang, J. Butler, and B. Tromberg. The Role of Diffuse Optical Spectroscopy in the Clinical Management of Breast Cancer. Disease Markers 19, 95-105 (20032004).
32. T. O. Mcbride, B. W. Pogue, S. Jiang, U. L. Osterberg, and K. D. Paulsen. A Parallel-detection Frequency-domain Near-infrared Tomography System for Hemoglobin Imaging of the Breast In Vivo. Review of Scientific Instruments 72, 1817-1824 (2001).

33. K. D. Paulsen and Jiang, H. Spatially Varying Optical Property Reconstruction Using a Finite Element Diffusion Equation Approximation. Med. Phys. 22, 691-701 (1995).

34. S. R. Arridge, M. Schweiger, M. Hiraoka, D. T. Delpy. A Finite Element Approach for Modeling Photon Transport in Tissue. Med. Phys. 20, 299-309 (1993).

35. T. O. McBride, B. W. Pogue, S. Jiang, U. L. Osterberg, K. D. Paulsen, and S. P. Poplack. Initial Studies of In Vivo Absorbing and Scattering Heterogeneity in Near-infrared Tomographic Breast Imaging. Opt. Lett. 26, 822-824 (2001).

36. H. J. van Staveren, C. J. M. Moes, J. van Marle, S. A. Prahl, and J. C. van Gemert. Light Scattering in Intralipid $-10 \%$ in the Wavelength Range of 400-1100nm. Applied Optics 30, 4507-4514 (1991).

37. J. R. Mourant, T. Fuselier, J. Boyer, T. M. Johnson, and I. J. Bigio. Predictions and Measurements of Scattering and Absorption Over Broad Wavelength Ranges in Tissue Phantoms. Applied Optics 36, 949 (1997).

38. X. Wang, B. W. Pogue, S. Jiang, X. Song, K. D. Paulsen, C. Kogel, S. P. Poplack, and W. A. Wells. Approximation of Mie Scattering Parameters in Near-infrared Tomography of Normal Breast Tissue In Vivo. Journal of Biomed Opt (in press) (2005).

39. J. R. Mourant, A. H. Hielscher, A. A. Eick, T. M. Johnson, and J. P. Freyer. Evidence of Intrinsic Differences in the Light Scattering Properties of Tumorigenic and Nontumorigenic Cells. Cancer Cytopathology 84, 366-374 (1998).

40. Breast Imaging Reporting and Data System (BIRADSTM). Reston[VA]: American College of Radiology (1998).

41. W. A. Wells, C. P. Daghlian, T. D. Tosteson, M. R. Grove, S. P. Poplack, S. Soho, and K. D. Paulsen. Analysis of the Microvasculature and Tissue Type Ratios in Benign and Malignant Breast Tissue. Analytical and Quantitative Cytology and Histology 26, 166-174 (2004).

42. S. Srinivasan, B. W. Pogue, H. Dehghani, S. Jiang, X. Song, and K. D. Paulsen. Improved Quantification of Small Objects in Nearinfrared Diffuse Optical Tomography. Journal of Biomed Opt. 9 , 1161-1171 (2004).

43. B. W. Pogue, S. Jiang, H. Dehghani, C. Kogel, S. Soho, S. Srinivasan, X. Song, S. P. Poplack, and K. D. Paulsen. Characterization of Hemoglobin, Water and NIR Scattering in Breast Tissue: Analysis of Inter-subject Variability and Menstrual Cycle Changes Relative to Lesions. J. Biomed. Opt. 9, 541-552 (2004).

44. S. P. Poplack, K. D. Paulsen, A. Hartov, P. M. Meaney, B. W. Pogue, T. D. Tosteson, M. R. Grove, S. K. Soho, and W. A. Wells. Electromagnetic Breast Imaging: Average Tissue Property Values in Women with Negative Clinical Findings. Radiology 231, 571580 (2004).

Date Received: May 2, 2005

Date Accepted: August 27, 2005 